\title{
THE PARABOLA THEOREM FOR CONTINUED FRACTIONS OVER A VECTOR SPACE
}

\author{
F. A. ROACH
}

ABSTRACT. In a recent paper, we defined a type of reciprocal for points of a real inner product space and considered continued fractions based on this reciprocal. These continued fractions were analogous to ordinary continued fractions in which each partial numerator is unity. In the present paper, we develop a type of continued fraction which is analogous to an ordinary continued fraction of the form in which each partial denominator is unity. The main result is a convergence theorem for such continued fractions which is a direct extension of a theorem by W. T. Scott and H. S. Wall (the Parabola Theorem).

1. Introduction. In [2] we introduced the notion of a continued fraction over a vector space. Following the notation used there, we let $S$ denote a real inner product space and $u$ denote a point of $S$ with unit norm. If $z$ is a point of $S$, we denote by $\bar{z}$ the point $2((z, u)) u-z$ and by $1 / z$ the point $\bar{z} /\|z\|^{2}$. (We assume that there is adjoined to $S$ a "point at infinity" with the usual conventions: $1 / 0=\infty, 1 / \infty=0$, etc.) Making use of this type of reciprocal, we considered continued fractions of the form

$$
b_{0}+\frac{1}{b_{1}}+\frac{1}{b_{2}}+\frac{1}{b_{3}}+\cdots .
$$

In [3], Scott and Wall gave an important convergence theorem (the Parabola Theorem) for continued fractions of the type in which each partial denominator is unity. The purpose of this paper is to define a continued fraction over $S$ which is analogous to one of this form and to give a theorem which is a direct extension of the Parabola Theorem. This is accomplished by a generalization of linear fractional transformations based on the observation that $T$ is a linear fractional transformation if and only if there exists a finite sequence $b_{0}, b_{1}$, $b_{2}, \cdots, b_{n}$, with each term a complex number, such that for every complex number $z, T(z)$ is

$$
b_{0}+\frac{1}{b_{1}}+\frac{1}{b_{2}}+\cdots+\frac{1}{b_{n}+z} .
$$

Received by the editors September 2, 1969.

AMS 1969 subject classifications. Primary 4012, 3025.

Key words and phrases. Continued fractions. 
Before proceeding, we call attention to two facts: (1) If $u$ is the unit vector with first coordinate 1 in either $E^{2}, E^{4}$, or $E^{8}$, then $1 / z$ reduces to the ordinary reciprocal for complex numbers, quaternions, or Cayley numbers, respectively. Thus, the results of this paper, as well as those of [2], hold true in those settings. (2) Every two dimensional subspace of $S$ which contains $u$ may be regarded as being the complex plane with $u$ corresponding to unity insofar as the transformations $\bar{z}$ and $1 / z$ are concerned.

2. Extended linear fractional transformation. Suppose that $T$ is an ordinary linear fractional transformation. Then there exist complex numbers $a, b, c$, and $d$ such that $a d-b c=1$ and $T(z)$ $=(a z+b) /(c z+d)$ for every complex number $z$. If $c \neq 0$, and in (1.2) we take $n=2, b_{0}=(a-1) / c, b_{1}=c$, and $b_{2}=(d-1) / c$, it reduces to $T(z)$. If $c=0$, then $a \neq 0$ and

$$
\frac{1}{1 / a}+\frac{1}{-a}+\frac{1}{(1-b) / a}+\frac{1}{1}+\frac{1}{-1}+\frac{1}{1+z}
$$

reduces to $T(z)$. Of course, if each one of $b_{0}, b_{1}, \cdots, b_{n}$ is a complex number, (1.2) is a linear fractional transformation. These observations motivate the following consideration.

Let $M$ denote the set of all transformations $T$ defined on $S$ such that for some sequence $b_{0}, b_{1}, \cdots, b_{n}$, with each term a point of $S$, $T(z)$ is given by (1.2) for every point $z$ of $S$. Clearly $M$ is closed under composition and since $1 /(1 / z)=z$, we see that each element of $M$ has an inverse in $M$. In fact, the inverse of (1.2) is

$$
-b_{n}+\frac{1}{-b_{n-1}}+\cdots+\frac{1}{-b_{0}+z} .
$$

Thus we have the following result.

\section{Theorem 1. The set $M$ forms a group under composition.}

The theorem below, which is analogous to the familiar statement about circles and lines, proves to be quite useful.

Theorem 2. Let $L$ denote the set of all hyperplanes and spheres in $S$. If $T$ is a transformation in $M$ and $K$ an element of $L$, then $T(K)$ is in $L$.

Since the elements of $M$ are composed of transformations of the form $b+z$ and $1 / z$, we need only consider transformations of this type. Clearly, if $T$ is a translation, i.e. of the form $b+z$, and $K$ is in $L$, then $T(K)$ is in $L$. Thus, it suffices to show that if $K$ is in $L$, then $1 / K-$ the image of $K$ under $1 / z-$ is in $L$. 
Suppose that $K$ is a hyperplane which contains 0 . Then for some point $P \neq 0, K$ is the set of all points $z$ such that $\|z-P\|=\|z+P\|$. Making use of the properties of the inner product and the fact that $((\bar{x}, \bar{y}))=((x, y))$, we see that this implies that

$$
\|1 / z-1 / P\|=\|1 / z+1 / P\| \text {. }
$$

Thus, $1 / K$ is a hyperplane. (We assume that $\infty$ belongs to every hyperplane.)

Now suppose that $c$ is a point and $r$ is a positive number. We will show that when $\|z-c\|=r$, then either

$$
\|1 / z-1 / c\|-\|1 / z\|
$$

or else

$$
\left\|1 / z+\bar{c} /\left(r^{2}-\|c\|^{2}\right)\right\|=r /\left|r^{2}-\|c\|^{2}\right|
$$

accordingly as $\|c\|$ is or is not $r$.

Consider first the case in which $\|c\|=r$. If $\|z-c\|=r$, we have that $\|z\|^{2}-2((z, c))=0$ and therefore,

$$
((c, c)) /\|c\|^{4}-2((z, c)) /\left(\|z\|^{2}\|c\|^{2}\right)=0 .
$$

This implies that $((1 / c, 1 / c))-2((1 / z, 1 / c))=0$ and hence, (2.1). Keeping in mind that $1 /(1 / z)=z$, we have not only that if $K$ is a sphere containing 0 , then $1 / K$ is a hyperplane, but also that if $K$ is a hyperplane which does not contain 0 , then $1 / K$ is a sphere.

Now suppose that $\|c\| \neq r$. From $\|z-c\|=r$, we obtain $((z, z))$ $-2((z, c))+((c, c))=r^{2}$ and hence

$$
4((z, c))^{2} /\|z\|^{2}-4((z, c))((\bar{z}, \bar{c})) /\|z\|^{2}-4((z, c))+\|\bar{z}+\bar{c}\|=r^{2} .
$$

This expression implies that $\left\|(1 / z)\left[2((z, c))-\|z\|^{2}\right]-\bar{c}\right\|=r$, which may be reduced to (2.2).

3. An extension of the Parabola Theorem. In order to simplify the statement and proof of the theorem of this section, we introduce some additional notation. For every point $a$ of $S$, we denote the point

$$
\frac{1}{-1 / a}+\frac{1}{a}+\frac{1}{(-1 / a)-u}
$$

by $a^{2}$. In case $S=E^{2}, E^{4}$, or $E^{8}$ with $u$ the unit vector having first coordinate 1 , the expression

$$
\frac{1}{-1 / a}+\frac{1}{a}+\frac{1}{(-1 / a)-b}
$$


reduces to the product $a b a$ for complex numbers, quaternions, or Cayley numbers, respectively. In view of this, we would expect that if $v$ is a unit vector such that $((u, v))=0$ and $x$ and $y$ are numbers, then $(x u+y v)^{2}=\left(x^{2}-y^{2}\right) u+(2 x y) v$. It is not difficult to establish that this is indeed the case.

TheOREM 3. Suppose that $S$ is complete, for $n=1,2,3, \cdots, a_{n}$ is a point of $S$ such that $\left\|a_{n}^{2}\right\|-\left(\left(a_{n}^{2}, u\right)\right) \leqq 1 / 2 ;$ and

$$
\begin{aligned}
T_{n}(z) & =\left(1 / a_{n}\right)+\frac{1}{-a_{n}}+\frac{1}{\left(1 / a_{n}\right)-\left(1 / a_{n}^{2}\right)-z} \quad \text { if } a_{n} \neq 0, \\
& =u \quad \text { if } a_{n}=0
\end{aligned}
$$

for every point $z$. Then $\lim T_{1} T_{2} \cdots T_{n}(u)$ exists and is finite as $n \rightarrow \infty$ if and only if either (i) some $a_{n}=0$ or else (ii) for $n=1,2,3, \cdots, a_{n} \neq 0$ and $\sum d_{n}$ diverges where $d_{1}=1$ and $d_{n+1}=1 /\left(d_{n}^{2}\left\|a_{n}\right\|\right)$ for $n=1,2$, $3, \cdots$.

Before giving a proof of this theorem, we call attention to the case in which $S=E^{2}$ and $u=(1,0)$. Here, for each $n, T_{n}(z)$ reduces to $1 /\left(1+a_{n}^{2} z\right)$ and the condition $\left\|a_{n}^{2}\right\|-\left(\left(a_{n}^{2}, u\right)\right) \leqq 1 / 2$ becomes $\left|a_{n}^{2}\right|$ $-\operatorname{Re}\left(a_{n}^{2}\right) \leqq 1 / 2$. The conclusion concerning $\lim T_{1} T_{2} \cdots T_{n}(u)$ is equivalent to the statement that the continued fraction

$$
\frac{1}{1}+\frac{a_{1}^{2}}{1}+\frac{a_{2}^{2}}{1}+\cdots
$$

converges if and only if (i) or (ii) holds true. Thus we see that this theorem includes the Parabola Theorem of Scott and Wall [3].

Throughout the following discussion, let $V$ denote the set of all points $z$ of $S$ such that $\|z-u\| \leqq 1$. If $K$ is a sphere or a spherical ball, we denote the radius of $K$ by $\operatorname{Rad}(K)$. Suppose that $n$ is a positive integer and $S^{\prime}$ is a two dimensional subspace of $S$ which contains both $a_{n}$ and $u$. If we denote $S^{\prime} \cap V$ by $V^{\prime}$ and regard $S^{\prime}$ as being the complex plane with $u$ corresponding to unity, we see that $T_{n}\left(V^{\prime}\right)$ is a subset of $V^{\prime}$ since $T_{n}(z)$ reduces to $1 /\left(1+a_{n}^{2} z\right)$ and $\left\|a_{n}^{2}\right\|-\left(\left(a_{n}^{2}, u\right)\right)$ $\leqq 1 / 2$. With the aid of Theorem 2 , we see that $T_{n}(V)$ is either the point $u$ or a spherical ball accordingly as $a_{n}$ is or is not 0 . In either case $T_{n}(V)$ is a subset of $V$. It is not difficult to see that if some $a_{n}=0$, then $\lim T_{1} T_{2} \cdots T_{n}(u)$ exists and is finite.

Now suppose that for $n=1,2,3, \cdots, a_{n} \neq 0$ and $\sum d_{n}$ diverges. For each $n$, let $V_{n}$ denote $T_{1} T_{2} \cdots T_{n}(V)$ and $r_{n}$ denote the radius of $V_{n}$. We have already seen that $V_{1}$ is a subset of $V$ and by a similar 
argument, one may show that if $K$ is a spherical ball which is a subset of $V$, then so also is $T_{n}(K)$. Therefore, for $n=1,2,3, \cdots, V_{n+1}$ is a subset of $V_{n}$. In order to establish that $\lim T_{1} T_{2} \cdots T_{n}(u)$ exists and is finite, it suffices to show that $\lim \left(r_{n}\right)=0$. We will accomplish this by showing that $r_{1}, r_{2}, r_{3}, \cdots$ is bounded by a certain sequence which has limit 0.

First, suppose that $K$ is a spherical ball with center $c$ and radius $r$ and that it is a subset of $V$. Let $B$ denote the boundary of $K$ (i.e. the sphere $\|z-c\|=r$ ) and let $n$ be a positive integer. For each point $z$, let $T(z)=1 /\left(1 / a-1 / a^{2}-z\right)$. We consider two cases: (1) $T(B)$ is a hyperplane and (2) $T(B)$ is a sphere.

In either case, we know that $1 /\left(-a_{n}+T(B)\right)$ is a sphere and that its radius is the same as the radius of $T_{n}(K)$. In case (1), with the aid of (2.1), we find that the point of $T(B)$ nearest 0 is $1 /(2 P)$ where $P=1 / T(c)$. Hence, the point of $-a_{n}+T(B)$ which is nearest 0 has norm

$$
\left(\left(-a_{n}, \bar{P} /\|P\|\right)\right)+1 /(2\|P\|) .
$$

But since in case (1) $\|P\|=r$, one half of this positive number is

$$
r /\left(\left\|a_{n}\right\|{ }^{2}\left[\dot{1} /\left\|a_{n}\right\|^{2}-2\left(\left(1 / \bar{a}_{n}, \bar{P}\right)\right)\right]\right)
$$

which reduces to

$$
r /\left(\left\|a_{n}\right\|^{2}\left[\left\|P-1 / a_{n}\right\|^{2}-r^{2}\right]\right) .
$$

Upon adding $\|P\|^{2}-r^{2}$ to the denominator of this expression and factoring, we have

$$
\operatorname{Rad} T_{n}(K)=r /\left(\left\|a_{n}\right\|^{2}\left[\left\|1 / a_{n}^{2}+c\right\|^{2}-r^{2}\right]\right) .
$$

In case (2), with the aid of (2.2), we find that

$$
\operatorname{Rad} T_{n}(K)=r\left|\|Q\|^{2}-r^{2}\right| /\left|\left\|\bar{Q}+a_{n}\left(\|Q\|^{2}-r^{2}\right)\right\|^{2}-r^{2}\right|
$$

where $Q=-1 / T(c)$. Making use of the properties of the inner product, this expression may be reduced to (3.2). Thus, in either case, (3.2) is an expression for the radius of $T_{n}(K)$.

Consider the function $f$ defined by $f(x)=\left(\left(x, a_{n}\right)\right)$ for every point $x$ such that $\|x\|=\|c\|$ and $((x, u))=((c, u))$. Let $S^{\prime}$ denote a two dimensional subspace of $S$ which contains both $a_{n}$ and $u$ and let $x^{\prime}$ denote a point of the domain of $f$ which lies in $S^{\prime}$. For every point $x$ in the domain of $f, f(x)$ is either $f\left(x^{\prime}\right), f\left(\bar{x}^{\prime}\right)$, or else between $f\left(x^{\prime}\right)$ and $f\left(\bar{x}^{\prime}\right)$. (This is easily seen from the fact that $\left|\left(\left(x, a_{n}\right)\right)\right| \leqq\|x\|\left\|a_{n}\right\|$ $=\|c\|\left\|a_{n}\right\|$ while one of $f\left(x^{\prime}\right)$ and $f\left(\bar{x}^{\prime}\right)$ is $\|c\|\left\|a_{n}\right\|$ and the other is $-\|c\|\left\|a_{n}\right\|$.) From this, we see that if $R(x)$ denotes 


$$
r /\left(\left\|a_{n}\right\|^{2}\left[\left\|1 / a_{n}^{2}+x\right\|^{2}-r^{2}\right]\right)
$$

for every point $x$ in the domain of $f$, then either $R\left(x^{\prime}\right) \geqq T_{n}(K)$ or else $R\left(\bar{x}^{\prime}\right) \geqq T_{n}(K)$. We will now proceed to name bounds on $r_{1}, r_{2}, r_{3}, \cdots$.

For every point $a$ of $S$, let

$$
\begin{array}{rlrl}
S(a ; z) & =1 / a+\frac{1}{-a}+\frac{1}{1 / a-1 / a^{2}-z} & \text { if } a \neq 0, \\
& =u \quad \text { if } a=0 .
\end{array}
$$

Of course if $a=a_{n}$, this transformation is $T_{n}$. Let $c_{1}$ denote $a_{1}$ and $S_{1}(z)$ denote $S\left(c_{1} ; z\right)$. Then $\operatorname{Rad} S_{1}(V)=\operatorname{Rad} T_{1}(V)$. Let $S^{\prime}$ denote a two dimensional subspace of $S$ which contains both $c_{1}$ and $u$ and let $c$ denote a point of $S^{\prime}$ such that $\|c\|=\left\|a_{2}\right\|$ and $((c, u))=\left(\left(a_{2}, u\right)\right)$. Let $c_{2}$ denote $c$ or $\bar{c}$ accordingly as $\operatorname{Rad} S_{1} S(c ; V)$ is or is not greater than $\operatorname{Rad} S_{1} S(\bar{c} ; V)$. We then have that

$$
\operatorname{Rad} S_{1} S_{2}(V) \geqq \operatorname{Rad} T_{1} T_{2}(V)
$$

where $S_{2}(z)$ is $S\left(c_{2} ; z\right)$. Now let $c^{\prime}$ denote a point of $S^{\prime}$ such that $\left\|c^{\prime}\right\|$ $=\left\|a_{3}\right\|$ and $\left(\left(c^{\prime}, u\right)\right)=\left(\left(a_{3}, u\right)\right)$. Let $c_{3}$ denote $c^{\prime}$ of $\bar{c}^{\prime}$ accordingly as $\operatorname{Rad} S_{2} S\left(c^{\prime} ; V\right)$ is or is not greater than $\operatorname{Rad} S_{2} S\left(\bar{c}^{\prime} ; V\right)$ and let $S_{3}(z)$ denote $S\left(c_{3} ; z\right)$. Notice that one of $S\left(c_{1} ; S_{2} S_{3}(V)\right)$ and $S\left(\bar{c}_{1} ; S_{2} S_{3}(V)\right)$ has a radius which is not less than the radius of $T_{1} T_{2} T_{3}(V)$. But $S_{2} S_{3}(V)$ is a subset of $S_{2}(V)$ and we have that

$$
\operatorname{Rad} S\left(c_{1} ; S_{2}(V)\right) \geqq \operatorname{Rad} S\left(\bar{c}_{1} ; S_{2}(V)\right) .
$$

Thus we see that

$$
\operatorname{Rad} S_{1} S_{2} S_{3}(V) \geqq \operatorname{Rad} T_{1} T_{2} T_{3}(V) .
$$

This process may be continued. Thus, there exists a sequence $c_{1}, c_{2}$, $c_{3}, \cdots$ with each term a point of $S^{\prime}$ and a sequence $S_{1}, S_{2}, S_{3}, \ldots$ such that, for $n=1,2,3, \cdots,\left\|c_{n}\right\|=\left\|a_{n}\right\|,\left(\left(c_{n}, u\right)\right)=\left(\left(a_{n}, u\right)\right)$, and

$$
\operatorname{Rad} S_{1} S_{2} \cdots S_{n}(V) \geqq \operatorname{Rad} T_{1} T_{2} \cdots T_{n}(V) .
$$

If we regard $S^{\prime}$ as being the complex plane with $u$ corresponding to unity, we have that $\left|c_{n}^{2}\right|-\operatorname{Re}\left(c_{n}^{2}\right) \leqq 1 / 2$. Let $V^{\prime}$ denote $S^{\prime} \cap V$. Paydon and Wall have shown in [1] that

$$
\lim \left[\operatorname{Rad} S_{1} S_{2} \cdots S_{n}\left(V^{\prime}\right)\right]=0 \quad \text { as } n \rightarrow \infty .
$$

From this it follows that $\lim \left[\operatorname{Rad} S_{1} S_{2} \cdots S_{n}(V)\right]=0$ and hence we conclude that when (ii) of Theorem 3 holds true, $\lim T_{1} T_{2} \cdots T_{n}(u)$ exists and is finite. 
We will now establish the necessity of the divergence of $\sum d_{n}$. Suppose that for $n=1,2,3, \cdots, a_{n} \neq 0$ and $\sum d_{n}$ converges. Let $b_{1}=u$ and, for $n=1,2,3, \cdots$, let

$$
b_{n+1}=\frac{1}{-b_{n}}+\frac{1}{-b_{n-1}}+\cdots+\frac{1}{-b_{1}}+\frac{1}{T_{1} T_{2} \cdots T_{n}(u)} .
$$

It is not difficult to see that for $n=1,2,3, \cdots$,

$$
T_{1} T_{2} \cdots T_{n}(u)=\frac{1}{b_{1}}+\frac{1}{b_{2}}+\cdots+\frac{1}{b_{n+1}} .
$$

We will now show that, for $n=1,2,3, \cdots, d_{n}=\left\|b_{n}\right\|$. Of course $d_{1}=\left\|b_{1}\right\|$ and we may establish directly that $d_{2}=\left\|b_{2}\right\|$. Suppose that $n$ is a positive integer greater than 2. From (3.4), $\left\|b_{n}\right\|=1 /\|x-y\|$ where

$$
x=\frac{1}{-b_{n-2}}+\cdots+\frac{1}{-b_{1}}+\frac{1}{T_{1} T_{2} \cdots T_{n-1}(u)}
$$

and

$$
y=\frac{1}{-b_{n-2}}+\cdots+\frac{1}{-b_{1}}+\frac{1}{T_{1} T_{2} \cdots T_{n-2}(u)} .
$$

Since $u,-a_{n-1}, 1 / a_{n-1}$, and $1 / a_{n-1}^{2}$ must all lie in some two dimensional subspace of $S$ which contains $u$, we have that $T_{n-1}(u)$ $=1 /\left(u+a_{n-1}^{2}\right)$. Expanding the expressions $T_{1} T_{2} \cdots T_{n-1}(u)$ and $T_{1} T_{2} \cdots T_{n-2}(u)$ by means of (3.1) and replacing $u$ by $1 / u$ in the second of these, we see that $y$ terminates with $1 / u$ and $x$ terminates with $1 /\left(u+1 /\left(1 / a_{n-1}^{2}\right)\right)$. In [2] we developed the following formula (equation (2.5))

where

$$
\left\|\left(\frac{1}{c_{1}}+\frac{1}{c_{2}}+\cdots+\frac{1}{c_{n}}\right)-\left(\frac{1}{c_{1}}+\frac{1}{c_{2}}+\cdots+\frac{1}{c_{n+1}}\right)\right\|
$$

$$
=\frac{1}{D_{n} D_{n+1}}
$$

$$
D_{k}=\left\|c_{k}\right\|\left\|c_{k-1}+1 / c_{k}\right\| \cdots\left\|c_{1}+\frac{1}{c_{2}}+\cdots+\frac{1}{c_{k}}\right\| .
$$

Applying this formula to the expression $1 /\|x-y\|$, we obtain

$$
\left\|b_{n}\right\|=A B
$$


where

$$
\begin{aligned}
& A=\left\|1 / a_{n-1}^{2}\right\|\left\|u+a_{n-1}^{2}\right\| \\
& \quad \cdots\left\|-b_{n-2}+\frac{1}{-b_{n-3}}+\cdots+\frac{1}{T_{n} T_{2} \cdots T_{n-1}(u)}\right\|
\end{aligned}
$$

and

$$
\begin{aligned}
& B=\|u\|\left\|1 / a_{n-2}-1 /^{2} a_{n-2}-u\right\| \\
& \cdots\left\|-b_{n-2}+\frac{1}{-b_{n-3}}+\cdots+\frac{1}{T_{1} T_{2} \cdots T_{n-2}(u)}\right\| .
\end{aligned}
$$

Notice that the last factor of $B$ is $1 /\left\|b_{n-1}\right\|$. Rearranging the factors of $A B$, we have that

$$
\left\|b_{n}\right\|=1 /\left(\left\|b_{n-1}\right\|\left\|a_{n-1}^{2}\right\|\right) f .
$$

We will now show that $f=1$. The last factor of $A$ may be written as

$$
\begin{aligned}
\|\left(\frac{1}{-b_{n-3}}+\cdots+\frac{1}{T_{1} T_{2} \cdots}\right. & \left.\frac{T_{n-2}\left(1 /\left(u+a_{n-1}^{2}\right)\right)}{\cdots}\right) \\
& -\left(\frac{1}{-b_{n-3}}+\cdots+\frac{1}{T_{1} T_{2} \cdots T_{n-2}(0)}\right) \|
\end{aligned}
$$

which, with the aid of (3.6), we may reduce to $1 / C D$ where

$$
C=\left\|u+a_{n-1}^{2}\right\| \cdots\left\|-b_{n-3}+\frac{1}{-b_{n-4}}+\cdots+\frac{1}{T_{1} T_{2} \cdots T_{n-1}(u)}\right\|
$$

and

$$
\begin{gathered}
D=\left\|1 / a_{n-2}-1 / a_{n-2}^{2}\right\|\left\|-a_{n-2}+1 /\left(1 / a_{n-2}-1 / a_{n-2}^{2}\right)\right\| \\
\cdots
\end{gathered}
$$

(In case $a_{n-2}=u$, we take the product of the first two factors of $D$ to be 1.) The product of the second through next to last factors of $A$ is $C$. The next to last factor of $B$ may be written as

$$
\begin{aligned}
& \|\left(\frac{1}{-b_{n-4}}+\cdots+\frac{1}{T_{1} T_{2} \cdots T_{n-3}\left(1 /\left(u+a_{n-2}^{2}\right)\right)}\right) \\
& -\left(\frac{1}{-b_{n-4}}+\cdots+\frac{1}{T_{1} T_{2} \cdots T_{n-3}(0)}\right) \|
\end{aligned}
$$


and then reduced to $1 / E F$ where

$$
E=\left\|u+a_{n-2}^{2}\right\| \cdots\left\|-b_{n-4}+\frac{1}{-b_{n-5}}+\cdots+\frac{1}{T_{1} T_{2} \cdots T_{n-2}(u)}\right\|
$$

and

$$
\begin{aligned}
F= & \left\|1 / a_{n-3}-1 / a_{n-3}^{2}\right\| \\
& \cdots\left\|-b_{n-4}+\frac{1}{-b_{n-5}}+\cdots+\frac{1}{T_{1} T_{2} \cdots T_{n-4}(u)}\right\| .
\end{aligned}
$$

Thus we have that $f=G /(D E F)$ where

$$
\begin{gathered}
G=\left\|1 / a_{n-2}-1 / a_{n-2}^{2}-u\right\|\left\|-a_{n-2}+1 /\left(1 / a_{n-2}-1 / a_{n-2}^{2}-u\right)\right\| \\
\cdots\left\|-b_{n-4}+\frac{1}{-b_{n-5}}+\cdots+\frac{1}{T_{1} T_{2} \cdots T_{n-2}(u)}\right\| .
\end{gathered}
$$

However, the product of the first two factors of $G$ is $\left\|u+a_{n-2}^{2}\right\| /\left\|a_{n-2}\right\|$, while the product of the first two factors of $D$ is $1 /\left\|a_{n-2}\right\|$. From this we see that $G=\left(1 /\left\|a_{n-2}\right\|\right) E$. The product of the first and third factors of $D$ is $\left\|u+a_{n-3}^{2}\right\| /\left\|a_{n-3}\right\|$. If we assume that $\left\|b_{n-2}\right\|=1 /\left(\left\|a_{n-3}^{2}\right\|\left\|b_{n-3}\right\|\right)$ and notice that the last factor of $F$ is $1 /\left\|b_{n-3}\right\|$ while the last factor of $D$ is $1 /\left\|b_{n-2}\right\|$, we see that $f=1 / M N$ where

$$
\begin{aligned}
M= & \left\|u+a_{n-3}^{2}\right\|\left\|1 / a_{n-4}-1 / a_{n-4}^{2}-1 /\left(u+a_{n-3}^{2}\right)\right\| \\
& \cdots\left\|-b_{n-4}+\frac{1}{-b_{n-5}}+\cdots+\frac{1}{T_{1} T_{2} \cdots T_{n-3}(u)}\right\|
\end{aligned}
$$

and

$$
\begin{aligned}
& N=\left\|1 / a_{n-4}-1 / a_{n-4}^{2}-u\right\| \\
& \quad \cdots\left\|-b_{n-5}+\frac{1}{-b_{n-6}}+\cdots+\frac{1}{T_{1} T_{2} \cdots T_{n-4}(u)}\right\| .
\end{aligned}
$$

If we compare $M N$ and $A B$, we conclude that by induction it follows that $f=1$.

Thus, we have that for $n=1,2,3, \cdots,\left\|b_{n+1}\right\|=1 /\left(\left\|a_{n}^{2}\right\|\left\|b_{n}\right\|\right)$, so that $d_{n}=\left\|b_{n}\right\|$. Theorem 1 of [2] states that if $\sum\left\|b_{n}\right\|$ converges, then

$$
\frac{1}{b_{1}}+\frac{1}{b_{2}}+\frac{1}{b_{3}}+\cdots
$$

diverges. This concludes the proof of Theorem 3 . 


\section{REFERENCES}

1. J. F. Paydon and H. S. Wall, The continued fraction as a sequence of linear transformations, Duke Math. J. 9 (1942), 360-372. MR 3, 297.

2. F. A. Roach, Continued fractions over an inner product space, Proc. Amer. Math. Soc. 24 (1970), 576-582.

3. W. T. Scott and H. S. Wall, A convergence theorem for continued fractions, Trans. Amer. Math. Soc. 47 (1940), 155-172. MR 1, 217.

University of Georgia, Athens, Georgia 30601 